\title{
Bow Shocks from Radio Pulsars: Observations of the Guitar Nebula
}

\author{
Shami Chatterjee and James M. Cordes \\ Astronomy and Space Sciences, Cornell University, Ithaca, NY 14853
}

\begin{abstract}
We present optical observations and radio non-detections of the bow shock nebula associated with the pulsar B2224+65 (the "Guitar Nebula"), and fit an analytic model to the observed bow shock to estimate its inclination and constrain other parameters (distance, pulsar velocity, ISM density). We also test scaling laws for bow shock parameters.
\end{abstract}

\section{Optical Observations of the Guitar Nebula}

The Guitar Nebula is a spectacular bow shock produced by B2224+65, a high velocity pulsar $(\mu=182 \pm 4$ mas $/ \mathrm{yr}, D \sim 2 \mathrm{kpc}$ ). H $\alpha$ images using the $5 \mathrm{~m}$ Hale telescope at Palomar Observatory show a nebula length comparable to about 300 years of travel, and its limb-brightened intensity varies on scales $\leq 10^{\prime \prime}$ (Cordes, Romani, \& Lundgren 1993). High resolution images $\left(0.05^{\prime \prime}\right.$ per pixel) of the bright head of the bow shock $\left(\sim 10^{\prime \prime}\right)$ were obtained with narrow-band $\mathrm{H} \alpha$ filters, using WFPC2 on the Hubble Space Telescope.

There are exact analytic solutions for stellar wind bow shocks in the thinshell limit (Wilkin 1996), balancing ram pressures of the stellar wind and ambient medium and then using momentum conservation to find the shape of the shell. We adapt this model for finite shell thicknesses and fit to the observed emission in the HST image. The model parameters are inclination to the line of sight (LOS), position angle, and a dimensionless scale parameter $S$, which depends on distance $D$, ambient medium density $n_{H}$, and the true energy loss rate $\dot{E}$ (where $\dot{E}_{I_{45}}$ is the spin-down energy loss rate assuming a moment of inertia of $\left.10^{45} \mathrm{gm}-\mathrm{cm}^{2}\right)$ :

$$
S=\left[\frac{\dot{E} / \dot{E}_{I_{45}}}{D_{\mathrm{kpc}}^{4} n_{H}}\right]^{1 / 2}
$$

A least-squares procedure gives the following best fit values: inclination to the LOS $=30^{\circ} ; S=10$. This constrains the density, distance, and projected velocity $V_{\perp}$, as shown in Table 1 , and implies that the true velocity of the pulsar is as high as twice the projected velocity.

It should be noted that the scale factor and inclination are correlated: as the size of the nebula is reduced due to projection, the scale increases (i.e. the distance decreases and/or the ISM density falls) to match the angular size on the sky. We also do not include uncertainties in neutron star moment of inertia, period, period derivative, or proper motion, all of which are small compared to the distance uncertainty. 
Table 1. Possible combinations of $D, n_{H}$, and $V$ for $S=10^{\dagger}$

\begin{tabular}{cccc}
\hline$n_{H}\left(\mathrm{~cm}^{-3}\right)$ & $\mathrm{D}(\mathrm{kpc})$ & $\mathrm{V}_{\perp}(\mathrm{km} / \mathrm{sec})$ & $V_{\text {total }}(\mathrm{km} / \mathrm{sec})$ \\
\hline 1.0 & 0.32 & 280 & 560 \\
0.1 & 0.56 & 480 & 960 \\
0.01 & 1.0 & 860 & 1700 \\
0.006 & $2.0^{\ddagger}$ & 1720 & 3400 \\
\hline
\end{tabular}

$\ddagger$ The nominal distance from dispersion measure.

† Other constraints from the $\mathrm{H} \alpha$ flux are under investigation.

\section{Radio Observations of the Guitar Nebula}

Radio observations were carried out with the NRAO Very Large Array in order to detect synchrotron emission from the relativistic pulsar wind or shockaccelerated particles, or possible thermal radiation from the interaction of the pulsar shock with a nearby filament. In observations of 2 to 3 hours, we obtain upper bounds $(3 \sigma)$ of $0.08,0.07$, and $0.08 \mathrm{mJy}$ for the emission at $1.4,4.9$, and $8.4 \mathrm{GHz}$ with 4,1 , and $2^{\prime \prime}$ resolutions respectively. At $1.4 \mathrm{GHz}$, this upper limit applies to positions offset from the detected pulsar, while at 4.9 and $8.4 \mathrm{GHz}$, the pulsar is not detected.

\section{Scaling Laws for Bow Shocks}

We derive the following simple scaling laws relating the unknown and observable parameters for bow shocks:

$$
\theta_{0} \propto\left[\frac{(\cos i)^{2}}{\sqrt{n_{H}}}\right]\left[\frac{\dot{E}}{D^{4} \mu^{2}}\right]^{1 / 2} ; F_{\alpha} \propto\left[\frac{X n_{H}}{(\cos i)^{5}}\right]\left(\theta_{0}^{2} \mu^{3} D^{3}\right)
$$

where the stand-off angle $\theta_{0}$ and the $\mathrm{H} \alpha$ flux $F_{\alpha}$ are observables, the LOS inclination $i$, the fraction of neutral atoms $X$, and the ISM density $n_{H}$ are unknowns, while proper motion $\mu$, distance $D$, and energy loss rate $\dot{E}$ are measurables.

There are several other pulsar wind nebulae besides the Guitar Nebula, two of which are detected in $\mathrm{H} \alpha$ emission. We use published parameters for B1957+20 and J0437-4715 along with B2224+65 and find good agreement with these scaling laws, given the $25-30 \%$ uncertainties in distances to these pulsars.

Acknowledgments. This research at Cornell University is supported in part by NSF grants AST 95-28391 and AST 99-19931.

\section{References}

Cordes, J. M., Romani, R. W., \& Lundgren, S. C. 1993, Nature, 362, 133

Wilkin, F. P., ApJ, 459, L31 